\title{
A Sequential Random Airy Type Problem of Fractional Order: Existence, Uniqueness and $\beta$-Differential Dependance
}

\author{
Yfrah Hafssa ${ }^{a}$, Zoubir Dahmani ${ }^{b}$ \\ a Laboratory of Pure and Applied Mathematics, University Abdelhamid Bni Badis of Mostaganem, Mostaganem, Algeria. \\ ${ }^{b}$ Laboratory of LPAM, University of Mostaganem, Mostaganem, Algeria.
}

\begin{abstract}
In this work, a new class of sequential random differential equations of Airy type is introduced. The existence and uniqueness criteria for stochastic process solutions for the introduced class are discussed. Some notions on $\beta$-differential dependance are also introduced. Then, new results on the $\beta$-dependance are discussed. At the end, some illustrative examples are discussed.
\end{abstract}

Keywords: Airy equation, mean square calculus, sequential random differential equation, stochastic process solution.

2010 MSC: 30C45, 39B72,39B82.

\section{Introduction}

The theory of fractional calculus has been distinguished in different fields of applied mathematics and many investigations have been stated as modeling, existence of solutions and various methods for solving fractional differential problems [2, 4, 5, 8, 9, 10, 15, 18, 20, 21, 22, 24, 27, 29, 33, 34].

Recently, the concept of fractional calculus and random differential equations have appeared as important and interesting subjects; this new random fractional theory has become very interesting to many researchers. To cite some papers related to this subject, we cite [1, 36, 19, 38, 39].

To investigate the theory of fractional differential equations with randomness, we have to use the mean

Email addresses: hafssa2014yfrah@gmail.com (Yfrah Hafssa), zzdahmani@yahoo.fr (Zoubir Dahmani) 
square calculus because of its importance for stochastic processes, see [16, 17, 31].

Many of random problems have been formulated by the Airy equation (and its solutions called Airy functions) which is given by

$$
Z^{\prime \prime}-t Z=0, \quad t \in \mathbb{R} .
$$

In [11], the authors have been concerned with the initial value problems for space-time-fractional Airy problem given by:

$$
\frac{\partial^{\alpha} u\left(x_{1}, t\right)}{\partial t^{\alpha}}=\frac{\partial^{\beta} u\left(x_{1}, t\right)}{\partial x_{1}^{\beta}}, 0<\alpha \leq 1,2<\beta \leq 3, x_{1} \in \mathbb{R},
$$

with $u\left(x_{1}, 0\right)=\frac{1}{6} x_{1}^{\beta}$.

In [30], M.D. Ovidio and E. Orsingher have expressed the law of the stable process $H^{v}(t), t>0$ in terms of Airy functions.

In [28], the authors have been concerned with the M-Wright function in time-fractional diffusion process and they have shown that the auxiliary functions can be expressed in terms of the Airy functions.

An example from quantum mechanics is given in the paper [26] where the exact solution of Schrodinger equation, for the motion of a particle in a homogeneous external field, can be expressed in terms of the Airy functions. Solutions of the Schrodinger equation involving the Airy functions are also given in [35].

For some other applications of Airy equations (and Airy functions) in elasticity theory, fluid mechanics and quantum physics, the reader is invited to see the research works [3, 6, 23, 25].

We cite also the paper [7], where the authors have studied the following random fractional initial value problem of Airy type:

$$
\left\{\begin{aligned}
\left({ }^{c} \mathbf{D}_{0_{+}}^{\alpha} Y\right)(t)-B t^{\beta} Y(t) & =0, t>0, n-1<\alpha \leq n, \beta>0 \\
Y^{(j)}(0) & =A_{j}, j=0,1, \ldots, n-1 .
\end{aligned}\right.
$$

Motivated by the above works, in the present paper we shall study a very important class of random fractional problem that generalizes the classical Airy-type differential equations both in the random and in the fractional senses. Specifically, we will deal with the following sequential random fractional generalized Airy-type problem:

$$
\left\{\begin{aligned}
\mathbf{D}^{\alpha_{1}} \cdots \mathbf{D}^{\alpha_{n}} Y(t) & =a_{1} A_{1} f_{1}(t, Y(t))+a_{2} A_{2} f_{2}\left(t, \mathbf{D}^{\beta} Y(t)\right)+a_{3} A_{3} f_{3}\left(t, \mathbf{I}^{\rho} Y(t)\right), \\
X_{0} & =Y(0), \\
X_{i} & =Y^{\left(\alpha_{i}+1\right)}(0), i=1, \ldots, n-1, n \in \mathbb{N}^{*} \\
t \in J=[0, T] & \left.\left., \quad \alpha_{i} \in\right] 0,1\right], 0<\beta<1,0<\rho,
\end{aligned}\right.
$$

where: $\mathbf{D}^{(\cdot)}$ represents the mean square derivative in the sense of Caputo, $Y(\cdot)$ is a second random function, $f_{i}: J \times \mathbb{L}_{2}(\Omega) \rightarrow \mathbb{L}_{2}(\Omega), i=1,2,3, X_{i}$ are second random variables $i=0, \ldots, n-1$, and $A_{1}, A_{2}, A_{3}$ are also second random variables. $a_{1}, a_{2}, a_{k}$ are real positive numbers.

It is to note that if $n=2, \alpha_{1}=\alpha_{2}=1, a_{2}=a_{3}=0$, then we obtain the standard Airy equation.

Under some other considerations on the input data of (2), we can obtain the generalized Airy problem of [7]. These are two reasons that have motivated the study of the above problem. And, to the best of our knowledge, there is no paper dealing with such random Airy type problem.

This paper is organized as follows: In section 2, we recall some definitions and lemmas that we need in the rest of the paper. In section 3, we present our main results for problem (2). Section 4 is devoted to introduce new concepts on random data dependence. In section 5 , we provide some examples of applications to illustrate our theoretical results. At the end, a conclusion follows.

\section{Basic Concepts}

Let $J:=[0, T]$ and consider a complete probability space $(\Omega, S, P)$. Over this space, we consider the stochastic process of order two $Y(t ; \omega)=\{Y(t), t \in J, \omega \in \Omega\}$ that satisfies $\left.E\left(Y^{2}(t)\right)<\infty, t \in J\right)$. 
Then, we consider:

$(1:) \mathbb{L}_{2}(\Omega)$ the Banach space of random variables $Y(t): \Omega \rightarrow \mathbb{R} ; E\left(Y^{2}\right)<\infty$, and

(2:) $\mathcal{C}=\mathcal{C}\left(J, \mathbb{L}_{2}(\Omega)\right)$ the set of all second order stochastic processes which are mean square continuous over $J$. This set is a Banach space equipped with the following norm:

$$
\|Y\|_{\mathcal{C}}=\sup _{t \in J}\|Y(t)\|_{2}, \text { where, }\|Y(t)\|_{2}=\left(E\left(Y^{2}(t)\right)\right)^{\frac{1}{2}}
$$

We recall the following definitions, see [12, 13, 14, 16].

Definition 2.1. Let $Y(t) \in \mathcal{C}, t \in J, \beta>0$. The mean square Riemann-Liouville integral of $Y$ of $\beta$-order is given by:

$$
\mathbf{I}^{\beta} Y(t):=\int_{0}^{t} \frac{(t-s)^{\beta-1}}{\Gamma(\beta)} Y(s) d s, t \in J .
$$

Definition 2.2. Let $Y(t) \in \mathcal{C}, t \in J, \beta>0, \beta \in] n-1, n], n=[\beta]+1, n \in \mathbb{N}^{*}$. The mean square Caputo derivative of $Y$ is given by

$$
\mathbf{D}^{\beta} Y(t):=\mathbf{I}^{n-\beta} Y^{(n)}(t) .
$$

Lemma 2.1. Let $\beta>0$. The solutions of $\mathbf{D}^{\beta} Y(t)=0$ are the following:

$$
Y(t)=C_{0}+C_{1} t+\cdots+C_{n-1} t^{n-1},
$$

with $C_{i} \in \mathbb{R}, i=0,1, \ldots, n-1,(n=[\beta]+1)$.

In view of this lemma, we can easily confirm that

$$
\mathbf{I}^{\beta} \mathbf{D}^{\beta} Y(t)=Y(t)+C_{0}+C_{1} t+\cdots+C_{n-1} t^{n-1} .
$$

\section{Existence and Uniqueness Criteria}

We begin our main result by proving the following random integral lemma.

Lemma 3.1. The random differential problem (2) has the following random integral representation

$$
\begin{aligned}
& Y(t)=\sum_{i=1}^{n-1} \frac{t^{\sum_{i=i+1}^{n} \alpha_{i}}}{\Gamma\left(\sum_{i=i+1}^{n} \alpha_{i}+1\right)} X_{i}+X_{0} \\
& +\int_{0}^{t} \frac{(t-s)^{\sum_{i=1}^{n} \alpha_{i}-1}}{\Gamma\left(\sum_{i=1}^{n} \alpha_{i}\right)}\left(a_{1} A_{1} f_{1}(s, Y(s))+a_{2} A_{2} f_{2}\left(s, \mathbf{D}^{\beta} Y(s)\right)+a_{3} A_{3} f_{3}\left(s, \mathbf{I}^{\rho} Y(s)\right)\right) d s .
\end{aligned}
$$

Proof. To prove the result, we begin by considering the following homogenous linear differential problem:

$$
\mathbf{D}^{\alpha_{1}} \cdots \mathbf{D}^{\alpha_{n}} Y(t)=W(t)
$$

where, $W(t):=a_{1} A_{1} f_{1}(t, Y(t))+a_{2} A_{2} f_{2}\left(t, \mathbf{D}^{\beta} Y(t)\right)+a_{3} A_{3} f_{3}\left(t, \mathbf{I}^{\rho} Y(t)\right)$.

Applying the mean square Riemann-Liouville integral of order $\alpha_{1}$, to (4), we can write

$$
\mathbf{D}^{\alpha_{2}} \cdots \mathbf{D}^{\alpha_{n}} Y(t)=\gamma_{1}+\mathbf{I}^{\alpha_{1}} W(t)
$$

Again, thanks to the square Riemann-Liouville integral of order $\alpha_{2}$, we can state that

$$
\mathbf{D}^{\alpha_{3}} \cdots \mathbf{D}^{\alpha_{n}} Y(t)=\gamma_{2}+\mathbf{I}^{\alpha_{2}} \gamma_{1}+\mathbf{I}^{\alpha_{1}+\alpha_{2}} W(t) .
$$

Consequently,

$$
\mathbf{D}^{\alpha_{3}} \cdots \mathbf{D}^{\alpha_{n}} Y(t)=\gamma_{2}+\frac{t^{\alpha_{2}}}{\Gamma\left(\alpha_{2}+1\right)} \gamma_{1}+\mathbf{I}^{\alpha_{1}+\alpha_{2}} W(t) .
$$


Using the same arguments as before, we get the following formula

$$
Y(t)=\sum_{i=1}^{n-1} \frac{t^{\sum_{i=i+1}^{n} \alpha_{i}}}{\Gamma\left(\sum_{i=i+1}^{n} \alpha_{i}+1\right)} \gamma_{i}+\gamma_{n}+\mathbf{I}^{\sum_{i=1}^{n} \alpha_{i}} W(t),
$$

where, $\gamma_{i} \in \mathbb{R}, i=1 \ldots, n$.

For $t=0$, in (8) we have

$$
Y(0)=\gamma_{n}
$$

and by the first initial condition in (2), we get $\gamma_{n}=X_{0}$. By differentiating of (8) $\alpha_{i+1}$-times for $i=1, \ldots, n-1$, and by taking $t=0$, we obtain

$$
\begin{gathered}
Y^{\left(\alpha_{n}\right)}(0)=\gamma_{n-1}, \\
\vdots \\
Y^{\left(\alpha_{2}\right)}(0)=\gamma_{1} .
\end{gathered}
$$

Also, we can see that

$$
\begin{gathered}
\gamma_{1}=X_{1}, \\
\vdots \\
\gamma_{n-1}=X_{n-1} .
\end{gathered}
$$

Substituting $\gamma_{i}, i=0, \ldots, n-1$, in (8), we get the desired representation. The proof is thus achieved.

Let now consider the Banach space defined by:

$$
F:=\left\{Y \in \mathcal{C}, \mathbf{D}^{\beta} Y \in \mathcal{C}\right\},
$$

which is equipped with the norm

$$
\|Y\|_{F}=\max \left(\|Y\|_{\mathcal{C}},\left\|\mathbf{D}^{\beta} Y\right\|_{\mathcal{C}}\right) .
$$

Let also introduce the random integral operator $H: F \rightarrow F$ :

$$
\begin{aligned}
& H Y(t)=\sum_{i=1}^{n-1} \frac{t^{\sum_{i=i+1}^{n} \alpha_{i}}}{\Gamma\left(\sum_{i=i+1}^{n} \alpha_{i}+1\right)} X_{i}+X_{0} \\
& +\int_{0}^{t} \frac{(t-s)^{\sum_{i=1}^{n} \alpha_{i}-1}}{\Gamma\left(\sum_{i=1}^{n} \alpha_{i}\right)}\left(a_{1} A_{1} f_{1}(s, Y(s))+a_{2} A_{2} f_{2}\left(s, \mathbf{D}^{\beta} Y(s)\right)+a_{3} A_{3} f_{3}\left(s, \mathbf{I}^{\rho} Y(s)\right)\right) d s .
\end{aligned}
$$

To facilitate the fastidious calculation, we consider the following notations and assumptions:

(H1) : There are three real positive numbers $K_{1}, K_{2}, K_{3}>0$, such that for all $Y_{1}, Y_{2} \in \mathbb{L}_{2}(\Omega), t \in J$, the following inequalities are valid:

$$
\begin{aligned}
& \left\|f_{1}\left(t, Y_{1}\right)-f_{1}\left(t, Y_{2}\right)\right\|_{2} \leq K_{1}\left\|Y_{1}-Y_{2}\right\|_{2}, \\
& \left\|f_{2}\left(t, Y_{1}\right)-f_{2}\left(t, Y_{2}\right)\right\|_{2} \leq K_{2}\left\|Y_{1}-Y_{2}\right\|_{2}, \\
& \left\|f_{3}\left(t, Y_{1}\right)-f_{3}\left(t, Y_{2}\right)\right\|_{2} \leq K_{3}\left\|Y_{1}-Y_{2}\right\|_{2} .
\end{aligned}
$$

(H2) : There exist three positive real numbers $0 \leq r_{1}, r_{2}, r_{3}$, such that

$$
\begin{aligned}
\left\|f_{1}(t, 0)\right\|_{2} & \leq r_{1}, \\
\left\|f_{2}(t, 0)\right\|_{2} & \leq r_{2}, \\
\left\|f_{3}(t, 0)\right\|_{2} & \leq r_{3} .
\end{aligned}
$$




$$
\begin{aligned}
\rho & =\sum_{i=1}^{n-1} \frac{T^{\sum_{i=i+1}^{n} \alpha_{i}}}{\Gamma\left(\sum_{i=i+1}^{n} \alpha_{i}+1\right)}\left\|X_{i}\right\|_{2}+\left\|X_{0}\right\|_{2}, \\
\rho_{1} & =\sum_{i=1}^{n-1} \frac{T^{\sum_{i=i+1}^{n} \alpha_{i}-\beta}}{\Gamma\left(\sum_{i=i+1}^{n} \alpha_{i}-\beta+1\right)}\left\|X_{i}\right\|_{2}, \\
\phi & =\frac{T^{\sum_{i=1}^{n} \alpha_{i}}}{\Gamma\left(\sum_{i=1}^{n} \alpha_{i}+1\right)}\left(a_{1}\left\|A_{1}\right\|_{2} r_{1}+a_{2}\left\|A_{2}\right\|_{2} r_{2}+a_{3}\left\|A_{3}\right\|_{2} r_{3}\right), \\
\phi_{1} & =\frac{T^{\sum_{i=1}^{n} \alpha_{i}}}{\Gamma\left(\sum_{i=1}^{n} \alpha_{i}+1\right)}\left(a_{1}\left\|A_{1}\right\|_{2} K_{1}+a_{2}\left\|A_{2}\right\|_{2} K_{2}+a_{3}\left\|A_{3}\right\|_{2} K_{3}\right), \\
\sigma & =\frac{T^{\sum_{i=1}^{n} \alpha_{i}-\beta}}{\Gamma\left(\sum_{i=1}^{n} \alpha_{i}-\beta+1\right)}\left(a_{1}\left\|A_{1}\right\|_{2} r_{1}+a_{2}\left\|A_{2}\right\|_{2} r_{2}+a_{3}\left\|A_{3}\right\|_{2} r_{3}\right), \\
\sigma_{1} & =\frac{T^{\sum_{i=1}^{n} \alpha_{i}-\beta}}{\Gamma\left(\sum_{i=1}^{n} \alpha_{i}-\beta+1\right)}\left(a_{1}\left\|A_{1}\right\|_{2} K_{1}+a_{2}\left\|A_{2}\right\|_{2} K_{2}+a_{3}\left\|A_{3}\right\|_{2} K_{3}\right) .
\end{aligned}
$$

Now, we prove the existence of a unique stochastic process solution for our above Airy type problem.

Theorem 3.1. Suppose satisfied the hypotheses (H.1) and (H.2). Then (2) has a unique stochastic process solution, under the condition that $R<1$, where

$$
R:=\max \left(\phi_{1}, \sigma_{1}\right)
$$

Proof. To prove this theorem, we shall consider an arbitrary real positive number $r$, such that

$$
r>\max \left(\frac{\rho+\phi}{1-\phi_{1}}, \frac{\rho_{1}+\sigma}{1-\sigma_{1}}\right) .
$$

We begin first by showing that $H B_{r} \subset B_{r}$, where

$$
B_{r}=\left\{Y \in F:\|Y\|_{F} \leq r\right\} .
$$

So, let $t \in J, Y \in B_{r}$. It is clear that by definition, we have

$$
\begin{aligned}
& \|H Y(t)\|_{2} \leq \sum_{i=1}^{n-1} \frac{t^{\sum_{i=i+1}^{n} \alpha_{i}}}{\Gamma\left(\sum_{i=i+1}^{n} \alpha_{i}+1\right)}\left\|X_{i}\right\|_{2}+\left\|X_{0}\right\|_{2} \\
& +\int_{0}^{t} \frac{(t-s)^{\sum_{i=1}^{n} \alpha_{i}-1}}{\Gamma\left(\sum_{i=1}^{n} \alpha_{i}\right)}\left(a_{1}\left\|A_{1}\right\|_{2}\left\|f_{1}(s, Y(s))\right\|_{2}+a_{2}\left\|A_{2}\right\|_{2}\left\|f_{2}\left(s, \mathbf{D}^{\beta} Y(s)\right)\right\|_{2}\right. \\
& \left.+a_{3}\left\|A_{3}\right\|_{2}\left\|f_{3}\left(s, \mathbf{I}^{\rho} Y(s)\right)\right\|_{2}\right) d s .
\end{aligned}
$$

Using both (H.1) and (H.2), we can state that

$$
\begin{aligned}
\left\|f_{1}(t, Y(t))-f_{1}(t, 0)+f_{1}(t, 0)\right\|_{2} & \leq\left\|f_{1}(t, Y(t))-f_{1}(t, 0)\right\|_{2}+\left\|f_{1}(t, 0)\right\|_{2} \\
& \leq K_{1}\|Y\|_{2}+r_{1} \leq K_{1}\|Y\|_{F}+r_{1} .
\end{aligned}
$$

With the same arguments, we get

$$
\begin{aligned}
\left\|f_{2}\left(t, \mathbf{D}^{\beta} Y(t)\right)-f_{2}(t, 0)+f_{2}(t, 0)\right\|_{2} & \leq\left\|f_{2}\left(t, \mathbf{D}^{\beta} Y(t)\right)-f_{2}(t, 0)\right\|_{2}+\left\|f_{2}(t, 0)\right\|_{2} \\
& \leq K_{2}\left\|\mathbf{D}^{\beta} Y\right\|_{2}+r_{2} \leq K_{2}\|Y\|_{F}+r_{2},
\end{aligned}
$$




$$
\begin{aligned}
\left\|f_{3}\left(t, \mathbf{I}^{\rho} Y(t)\right)-f_{3}(t, 0)+f_{3}(t, 0)\right\|_{2} & \leq\left\|f_{3}\left(t, \mathbf{I}^{\rho} Y(t)\right)-f_{3}(t, 0)\right\|_{2}+\left\|f_{3}(t, 0)\right\|_{2} \\
& \leq K_{3}\left\|\mathbf{I}^{\rho} Y\right\|_{2}+r_{3} \leq K_{3}\|Y\|_{F}+r_{3} .
\end{aligned}
$$

Therefore, it yields that

$$
\begin{aligned}
& \|H Y(t)\|_{2} \leq \sum_{i=1}^{n-1} \frac{T^{\sum_{i=i+1}^{n} \alpha_{i}}}{\Gamma\left(\sum_{i=i+1}^{n} \alpha_{i}+1\right)}\left\|X_{i}\right\|_{2}+\left\|X_{0}\right\|_{2} \\
& +\frac{T^{\sum_{i=1}^{n} \alpha_{i}}}{\Gamma\left(\sum_{i=1}^{n} \alpha_{i}+1\right)}\left(a_{1}\left\|A_{1}\right\|_{2}\left(K_{1}\|Y\|_{\mathcal{C}}+r_{1}\right)+a_{2}\left\|A_{2}\right\|_{2}\left(K_{2}\left\|\mathbf{D}^{\beta} Y\right\|_{\mathcal{C}}+r_{2}\right)\right. \\
& \left.+a_{3}\left\|A_{3}\right\|_{2}\left(K_{3}\left\|\mathbf{I}^{\rho} Y\right\|_{\mathcal{C}}+r_{3}\right)\right) .
\end{aligned}
$$

So, we obtain

$$
\begin{aligned}
& \|H Y\|_{\mathcal{C}} \leq \sum_{i=1}^{n-1} \frac{T^{\sum_{i=i+1}^{n} \alpha_{i}}}{\Gamma\left(\sum_{i=i+1}^{n} \alpha_{i}+1\right)}\left\|X_{i}\right\|_{2}+\left\|X_{0}\right\|_{2} \\
& +\frac{T^{\sum_{i=1}^{n} \alpha_{i}}}{\Gamma\left(\sum_{i=1}^{n} \alpha_{i}+1\right)}\left(a_{1}\left\|A_{1}\right\|_{2}\left(K_{1}\|Y\|_{F}+r_{1}\right)+a_{2}\left\|A_{2}\right\|_{2}\left(K_{2}\|Y\|_{F}+r_{2}\right)+a_{3}\left\|A_{3}\right\|_{2}\left(K_{3}\|Y\|_{F}+r_{3}\right)\right) \\
& \leq \rho+\phi+\phi_{1} r<r .
\end{aligned}
$$

On the other side, we can write

$$
\begin{aligned}
& \left\|\mathbf{D}^{\beta} H Y\right\|_{\mathcal{C}} \leq \sum_{i=1}^{n-1} \frac{T^{\sum_{i=i+1}^{n} \alpha_{i}-\beta}}{\Gamma\left(\sum_{i=i+1}^{n} \alpha_{i}-\beta+1\right)}\left\|X_{i}\right\|_{2} \\
& +\frac{T^{\sum_{i=1}^{n} \alpha_{i}-\beta}}{\Gamma\left(\sum_{i=1}^{n} \alpha_{i}-\beta+1\right)}\left(a_{1}\left\|A_{1}\right\|_{2}\left(K_{1}\|Y\|_{F}+r_{1}\right)+a_{2}\left\|A_{2}\right\|_{2}\left(K_{2}\|Y\|_{F}+r_{2}\right)+a_{3}\left\|A_{3}\right\|_{2}\left(K_{3}\|Y\|_{F}+r_{3}\right)\right) \\
& \leq \rho_{1}+\sigma+\sigma_{1} r<r .
\end{aligned}
$$

Thanks to 10 and 11 , we can deduce that

$$
\|H Y\|_{F} \leq r
$$

We have thus proved that $H B_{r} \in B_{r}$.

Now, we prove that $H$ is contractive.

Let $Y_{1}, Y_{2} \in F, t \in J$. We have

$$
\begin{aligned}
& H Y_{1}(t)-H Y_{2}(t)=\int_{0}^{t} \frac{(t-s)^{\sum_{i=1}^{n} \alpha_{i}-1}}{\Gamma\left(\sum_{i=1}^{n} \alpha_{i}\right)}\left(a_{1} A_{1}\left(f_{1}\left(s, Y_{1}(s)\right)-f_{1}\left(s, Y_{2}(s)\right)\right)\right. \\
& \left.+a_{2} A_{2}\left(f_{2}\left(s, \mathbf{D}^{\beta} Y_{1}(s)\right)-f_{2}\left(s, \mathbf{D}^{\beta} Y_{2}(s)\right)\right)+a_{3} A_{3}\left(f_{3}\left(s, \mathbf{I}^{\rho} Y_{1}(s)\right)-f_{3}\left(s, \mathbf{I}^{\rho} Y_{2}(s)\right)\right)\right) d s,
\end{aligned}
$$

which leads to

$$
\begin{aligned}
& \left\|H Y_{1}(t)-H Y_{2}(t)\right\|_{2} \leq \int_{0}^{t} \frac{(t-s)^{\sum_{i=1}^{n} \alpha_{i}-1}}{\Gamma\left(\sum_{i=1}^{n} \alpha_{i}\right)}\left(a_{1}\left\|A_{1}\right\|_{2}\left\|f_{1}\left(s, Y_{1}(s)\right)-f_{1}\left(s, Y_{2}(s)\right)\right\|_{2}\right. \\
& \left.+a_{2}\left\|A_{2}\right\|_{2}\left\|f_{2}\left(s, \mathbf{D}^{\beta} Y_{1}(s)\right)-f_{2}\left(s, \mathbf{D}^{\beta} Y_{2}(s)\right)\right\|_{2}+a_{3}\left\|A_{3}\right\|_{2}\left\|f_{3}\left(s, \mathbf{I}^{\rho} Y_{1}(s)\right)-f_{3}\left(s, \mathbf{I}^{\rho} Y_{2}(s)\right)\right\|_{2}\right) d s .
\end{aligned}
$$


Thanks to (H.1), we have the following estimate

$$
\begin{aligned}
& \left\|H Y_{1}-H Y_{2}\right\|_{\mathcal{C}} \leq \frac{T^{\sum_{i=1}^{n} \alpha_{i}}}{\Gamma\left(\sum_{i=1}^{n} \alpha_{i}+1\right)} \\
& \times\left(a_{1}\left\|A_{1}\right\|_{2}\left(K_{1}\left\|Y_{1}-Y_{2}\right\|_{\mathcal{C}}\right)+a_{2}\left\|A_{2}\right\|_{2}\left(K_{2}\left\|\mathbf{D}^{\beta} Y_{1}-\mathbf{D}^{\beta} Y_{2}\right\|_{\mathcal{C}}\right)+a_{3}\left\|A_{3}\right\|_{2}\left(K_{3}\left\|\mathbf{I}^{\rho} Y_{1}-\mathbf{I}^{\rho} Y_{2}\right\|_{\mathcal{C}}\right)\right) \\
& \leq \phi_{1}\left\|Y_{1}-Y_{2}\right\|_{F} .
\end{aligned}
$$

Some easy calculation will allow us to state that

$$
\begin{aligned}
& \left\|\mathbf{D}^{\beta}\left(H Y_{1}-H Y_{2}\right)\right\|_{\mathcal{C}} \leq \frac{T^{\sum_{i=1}^{n} \alpha_{i}-\beta}}{\Gamma\left(\sum_{i=1}^{n} \alpha_{i}-\beta+1\right)} \\
& \times\left(a_{1}\left\|A_{1}\right\|_{2}\left(K_{1}\left\|Y_{1}-Y_{2}\right\|_{\mathcal{C}}\right)+a_{2}\left\|A_{2}\right\|_{2}\left(K_{2}\left\|\mathbf{D}^{\beta} Y_{1}-\mathbf{D}^{\beta} Y_{2}\right\|_{\mathcal{C}}\right)+a_{3}\left\|A_{3}\right\|_{2}\left(K_{3}\left\|\mathbf{I}^{\rho} Y_{1}-\mathbf{I}^{\rho} Y_{2}\right\|_{\mathcal{C}}\right)\right) \\
& \leq \sigma_{1}\left\|Y_{1}-Y_{2}\right\|_{F} .
\end{aligned}
$$

The inequalities $(12)$ and $(13)$ allow us to say that

$$
\left\|H Y_{1}-H Y_{2}\right\|_{F} \leq \max \left(\phi_{1}, \sigma_{1}\right)\left\|Y_{1}-Y_{2}\right\|_{F} .
$$

At the end of this proof, we can conclude that problem (2) has a unique stochastic process solution on $J$.

\section{Random Data and $\beta$-Dependance}

Using the introduced norm of the above Banach space, we shall be concerned with introducing some random dependance definitions for the above fractional Airy type problem. Then, we prove some random variables data dependance results for the same problem.

To do this, we shall first consider the following auxiliary problem:

$$
\left\{\begin{aligned}
\mathbf{D}^{\alpha_{1}} \cdots \mathbf{D}^{\alpha_{n}} Y(t) & =a_{1} A_{1} f_{1}(t, Y(t))+a_{2} A_{2} f_{2}\left(t, \mathbf{D}^{\beta} Y(t)\right)+a_{3} A_{3} f_{3}\left(t, \mathbf{I}^{\rho} Y(t)\right), 0 \leq a_{i} \\
\tilde{X}_{0} & =Y(0) \\
\tilde{X}_{i} & =Y^{\left(\alpha_{i}+1\right)}(0), i=1, \ldots, n-1, n \in \mathbb{N}^{*} \\
t \in J=[0, T] & \left.\left., \quad \alpha_{i} \in\right] 0,1\right], 0<\beta<1,0<\rho
\end{aligned}\right.
$$

We introduce the following first definition.

Definition 4.1. The solution $Y$ of $(2)$ is continuously and $\beta$-differentially dependent on the random data $X_{i}, i=0, \ldots, n-1, n \in \mathbb{N}^{*}$, if

$$
\forall \epsilon>0, \exists \delta_{i}>0, i=0, \ldots, n-1, \text { such that }\left\|X_{i}-\tilde{X}_{i}\right\|_{2} \leq \delta_{i} \text { the inequality }\|Y-\tilde{Y}\|_{F} \leq \epsilon
$$

holds.

At this moment, we are able to present to the reader the following main result.

Theorem 4.1. Suppose that the conditions of Theorem 3.2 are valid. Then the solution of (2) is continuously and $\beta$-differentially dependent on $X_{i}, i=0, \ldots, n-1, n \in \mathbb{N}^{*}$.

Proof. Let $Y$ and $\tilde{Y}$ be the unique random solution of (2) and (14), where:

$$
\begin{aligned}
& \tilde{Y}(t)=\sum_{i=1}^{n-1} \frac{t^{\sum_{i=i+1}^{n} \alpha_{i}}}{\Gamma\left(\sum_{i=i+1}^{n} \alpha_{i}+1\right)} \tilde{X}_{i}+\tilde{X}_{0} \\
& +\int_{0}^{t} \frac{(t-s)^{\sum_{i=1}^{n} \alpha_{i}-1}}{\Gamma\left(\sum_{i=1}^{n} \alpha_{i}\right)}\left(a_{1} A_{1} f_{1}(s, \tilde{Y}(s))+a_{2} A_{2} f_{2}\left(s, \mathbf{D}^{\beta} \tilde{Y}(s)\right)+a_{3} A_{3} f_{3}\left(s, \mathbf{I}^{\rho} \tilde{Y}(s)\right)\right) d s .
\end{aligned}
$$


We have

$$
\begin{aligned}
& Y(t)-\tilde{Y}(t)=\sum_{i=1}^{n-1} \frac{t^{\sum_{i=i+1}^{n} \alpha_{i}}}{\Gamma\left(\sum_{i=i+1}^{n} \alpha_{i}+1\right)}\left(X_{i}-\tilde{X}_{i}\right)+\left(X_{0}-\tilde{X}_{0}\right) \\
& +\int_{0}^{t} \frac{(t-s)^{\sum_{i=1}^{n} \alpha_{i}-1}}{\Gamma\left(\sum_{i=1}^{n} \alpha_{i}\right)}\left(a_{1} A_{1}\left(f_{1}(s, Y(s))-f_{1}(s, \tilde{Y}(s))\right)+a_{2} A_{2}\left(f_{2}\left(s, \mathbf{D}^{\beta} Y(s)\right)-f_{2}\left(s, \mathbf{D}^{\beta} \tilde{Y}(s)\right)\right)\right. \\
& \left.+a_{3} A_{3}\left(f_{3}\left(s, \mathbf{I}^{\rho} Y(s)\right)-f_{3}\left(s, \mathbf{I}^{\rho} \tilde{Y}(s)\right)\right)\right) d s .
\end{aligned}
$$

So, we get

$$
\begin{aligned}
& \|Y(t)-\tilde{Y}(t)\|_{2} \leq \sum_{i=1}^{n-1} \frac{t^{\sum_{i=i+1}^{n} \alpha_{i}}}{\Gamma\left(\sum_{i=i+1}^{n} \alpha_{i}+1\right)}\left\|X_{i}-\tilde{X}_{i}\right\|_{2}+\left\|X_{0}-\tilde{X}_{0}\right\|_{2} \\
& +\int_{0}^{t} \frac{(t-s)^{\sum_{i=1}^{n} \alpha_{i}-1}}{\Gamma\left(\sum_{i=1}^{n} \alpha_{i}\right)}\left(a_{1}\left\|A_{1}\right\|_{2}\left\|f_{1}(s, Y(s))-f_{1}(s, \tilde{Y}(s))\right\|_{2}+a_{2}\left\|A_{2}\right\|_{2}\left\|f_{2}\left(s, \mathbf{D}^{\beta} Y(s)\right)-f_{2}\left(s, \mathbf{D}^{\beta} \tilde{Y}(s)\right)\right\|_{2}\right. \\
& \left.+a_{3}\left\|A_{3}\right\|_{2}\left\|f_{3}\left(s, \mathbf{I}^{\rho} Y(s)\right)-f_{3}\left(s, \mathbf{I}^{\rho} \tilde{Y}(s)\right)\right\|_{2}\right) d s .
\end{aligned}
$$

Consequently, we obtain

$$
\|Y-\tilde{Y}\|_{\mathcal{C}} \leq \sum_{i=1}^{n-1} \frac{T^{\sum_{i=i+1}^{n} \alpha_{i}}}{\Gamma\left(\sum_{i=i+1}^{n} \alpha_{i}+1\right)} \delta_{i}+\delta_{n}+\phi_{1}\|Y-\tilde{Y}\|_{F} .
$$

With the same arguments as before, we have

$$
\left\|\mathbf{D}^{\beta}(Y-\tilde{Y})\right\|_{\mathcal{C}} \leq \sum_{i=1}^{n-1} \frac{T^{\sum_{i=i+1}^{n} \alpha_{i}-\beta}}{\Gamma\left(\sum_{i=i+1}^{n} \alpha_{i}-\beta+1\right)} \delta_{i}+\sigma_{1}\|Y-\tilde{Y}\|_{F}
$$

By the inequalities (22) and (23), we get

$$
\|Y-\tilde{Y}\|_{F} \leq \max \left(\sum_{i=1}^{n-1} \frac{T^{\sum_{i=i+1}^{n} \alpha_{i}}}{\Gamma\left(\sum_{i=i+1}^{n} \alpha_{i}+1\right)} \delta_{i}+\delta_{n}, \sum_{i=1}^{n-1} \frac{T^{\sum_{i=i+1}^{n} \alpha_{i}-\beta}}{\Gamma\left(\sum_{i=i+1}^{n} \alpha_{i}-\beta+1\right)} \delta_{i}\right)+\max \left(\phi_{1}, \sigma_{1}\right)\|Y-\tilde{Y}\|_{F} .
$$

This leads to

$$
\|Y-\tilde{Y}\|_{F} \leq \frac{\max \left(\sum_{i=1}^{n-1} \frac{T^{\sum_{i=i+1}^{n} \alpha_{i}}}{\Gamma\left(\sum_{i=i+1}^{n} \alpha_{i}+1\right)} \delta_{i}+\delta_{n}, \sum_{i=1}^{n-1} \frac{T^{\sum_{i=i+1}^{n} \alpha_{i}-\beta}}{\Gamma\left(\sum_{i=i+1}^{n} \alpha_{i}-\beta+1\right)} \delta_{i}\right)}{1-R},
$$

where $R=\max \left(\phi_{1}, \sigma_{1}\right)$.

The proof is thus complete.

\section{Applications}

This section deals with two examples to review the main results by a numerical point of view.

Example 5.1. We consider the following initial value problem

$$
\begin{aligned}
\mathbf{D}^{0.7} \mathbf{D}^{0.4} Y(t)= & 1.5 A_{1} \frac{\cos Y(t)+Y(t)}{33\left(t^{2}+2\right)}+\sqrt{3} A_{2} \frac{\mathbf{D}^{\frac{1}{25}} Y(t)+\sin \mathbf{D}^{\frac{1}{25}} Y(t)}{t+31} \\
& +\frac{1}{2} A_{3} \frac{2 \cos \mathbf{I}^{\frac{3}{2}} Y(t)+2 \sin \mathbf{I}^{\frac{3}{2}} Y(t)}{\exp (\sqrt{t}+23)}
\end{aligned}
$$


such that $E\left(X_{0}^{2}\right)=1, E\left(X_{1}^{2}\right)=3, E\left(A_{1}^{2}\right)=4, E\left(A_{2}^{2}\right)=1, E\left(A_{3}^{2}\right)=16$, where $t \in J=[0,7]$.

We have $K_{1}=\frac{1}{66}, K_{2}=\frac{1}{31}, K_{3}=\frac{2}{\exp 23}, r_{1}=\frac{1}{33\left(t^{2}+2\right)}, r_{2}=0, r_{3}=\frac{2}{\exp (\sqrt{t}+23)}$.

We get $\rho=5.2515, \rho_{1}=3.9203, \phi=0.3694, \phi_{1}=0.8234, \sigma=0.3482, \sigma_{1}=0.7763$, and $R=\max \left(\phi_{1}, \sigma_{1}\right)=$ $0.8234<1$.

Thanks to Theorem 3.2, the problem 22 has a unique stochastic process solution on $J=[0,7]$.

Example 5.2. Consider the following problem

$$
\mathbf{D}^{0.6} \mathbf{D}^{0.6} \mathbf{D}^{0.9} Y(t)=0.75 A_{1} f_{1}(t, Y(t))+2 A_{2} f_{2}\left(t, \mathbf{D}^{\frac{2}{33}} Y(t)\right)+A_{3} f_{3}\left(t, \mathbf{I}^{3} Y(t)\right),
$$

such that $E\left(X_{0}^{2}\right)=2, E\left(X_{1}^{2}\right)=1, E\left(X_{2}^{2}\right)=5, E\left(A_{1}^{2}\right)=9, E\left(A_{2}^{2}\right)=16, E\left(A_{3}^{2}\right)=1$, where $t \in J=[0,5]$, and

$$
\begin{aligned}
& f_{1}(t, Y(t))=\frac{1}{2 t+43}(\sin Y(t)+\cos Y(t)), \\
& f_{2}\left(t, \mathbf{D}^{\frac{2}{23}} Y(t)\right)=\frac{1}{\sqrt{t}+\exp (27)}\left(\mathbf{D}^{\frac{2}{23}} Y(t)+\cos \mathbf{D}^{\frac{2}{23}} Y(t)\right), \\
& f_{3}\left(t, \mathbf{I}^{3} Y(t)\right)=\frac{\mathbf{I}^{3} Y(t)}{t^{3}+47} .
\end{aligned}
$$

We have $K_{1}=\frac{1}{43}, K_{2}=\frac{1}{\exp (27)}, K_{3}=\frac{1}{47}, r_{1}=\frac{1}{2 t+43}, r_{2}=\frac{1}{\sqrt{t}+\exp (47)}, r_{3}=0$.

Using our data, we find $\rho=19.7213, \rho_{1}=17.1148, \phi=0.6992, \phi_{1}=0.9835, \sigma=0.6718, \sigma_{1}=0.9450$, and $R=\max \left(\phi_{1}, \sigma_{1}\right)=0.9835<1$.

Then, by Theorem 4.2, the problem (23) is continuously and $\frac{2}{33}$-differentially dependent on $X_{i}, i=0,1,2$.

\section{Conclusion}

We have studied a class of random fractional problems using mean square calculus notions. The considered problem generalizes the classical Airy differential equation both in the random and in the fractional senses. We have established new sufficient conditions to prove the existence of a unique stochastic process solution. Some notions on $\beta$-differential dependance have also been introduced in the paper and new results on such dependance have been established. At the end, two illustrative examples have also been discussed. In the future, the Ulam-Hyers stability for problem (2) will be analysed, then it will be compared with the $\beta$-dependance results of the present paper. This paper is in progress...

\section{References}

[1] S. Abbas, N. Al Arifi, M. Benchohra, J. Graef, Random coupled systems of implicit Caputo-Hadamard fractional differential equations with multi-point boundary conditions in generalized Banach spaces, Dynamics Systems and Applications. 28(2) (2019) 329-350.

[2] B. Alqahtani, H. Aydi, E. Karapinar and V. Rakocevic, A solution for volterra fractional integral equations by hybrid contractions, Mathematics. 7(8) (2019) 694.

[3] S.S. Alshehri, Properties of Airy functions and application to the V-Shape potential, MECSJ. 15 (2018).

[4] D. Baleanu, S. Etemad and S. Rezapour, A hypbrid Caputo fractional modeling for thermostat with hybrid boundary value conditions, Boundary Value Problems. 64 (2020).

[5] D. Baleanu, S. Rezapour, A new study on the mathematical modelling of human liver with Caputo-Fabrizio fractional derivative, Chaos, Solitons and Fractals. 134 (2020) 109705.

[6] C. Burgos, J.C. Cortes, A. Debbouche, L. Villafuerte and R.J. Villanueva, Random fractional generalized Airy differential equations: a probabilistic analysis using mean square calculus, Applied Mathematics and Computation. 352 (2019) 15-29.

[7] C. Burgos, J.C. Corts, M.D. Rosello and R.J. Villanueva, Some tools to study random fractional differential equations and applications, Springer. (2020)

[8] Z. Dahmani and M.A. Abdellaouil, On a three point boundary value problem of arbitrary order, Journal of Interdisciplinary Mathematics. 19(5-6) (2016) 893-906.

[9] Z. Dahmani, M.A. Abdelaoui and M. Houas, Polynomial solutions for a class of fractional differential equations and systems, Journal of Interdisciplinary Mathematics. 21(3) (2018) 669-680.

[10] Z. Dahmani and L. Marouf, Numerical study of differential equation governing speech gestures with Caputo derivative, Journal of Interdisciplinary Mathematics. 16(4-5) (2013) 287-296. 
[11] D.B. Dhaigude, S.G. Jadhav, L.J. Mahmood, Solution of space time fractional partial differential equations by Adomian decomposition method, Bulletin of the Marathwada Mathematical Society. 15(1) (2014) 26-37.

[12] A.M.A. El-Sayed, The mean square Riemann-Liouville stochastic fractional derivative and stochastic fractional order differential equation, Math. Sei. Res. J. 9 (2005) 142-150.

[13] A.M.A. El-Sayed, On the stochastic fractional calculus operators, Journal of Fractional Calculus and Applications.6(1) (2015) 101-109.

[14] A.M.A. El- Sayed, F. Gaafar and M. El-Gendy, Continuous dependence of the solution of random fractional-order differential equation with nonlocal conditions, J. Fractional Differential Calculus. 7(1) (2017) 135-149.

[15] R. Gorenflo, F. Mainardi, Essentials of fractional calculus, Maphysto Center. (2000).

[16] F.M. Hafiz, The fractional calculus for some stochastic processes, Stoch. Anal. Appl. 22 (2004) 507-523.

[17] F.M. Hafiz, A.M.A. El-Sayed, and M.A. El-Tawil, On a stochastic fractional calculus, Frac. Calc; Appl. Anal.4 (2001) $81-90$.

[18] N. Heymans, I. Podlubny, Physical interpretation of initial conditions for fractional diffrential equations with ReimannLiouville fractional derivatives, Rheologica Acta. 45(5) (2006) 765-771.

[19] V. Ho, Random fractional functional differential equations, International journal of nonlinear analysis and applications. $7(2)$ (2016) 253-267.

[20] J.S. Jacob, J.H. Priya, A. Karthika, Applications of fractional calculus in science and engineering, JCR. 7(13) (2020) 4385-4394.

[21] E. Karapinar, A. Fulga, M. Rashid, L. Shahid and H. Aydi, Large contractions on quasi-metric spaces with an applications to nonlinear fractional differential equations, Mathematics. 7(5) (2019) 7050444.

[22] H.M. Kilbas, H.M. Srivastava, J.J. Trujillo, Theory and applications of fractional differential equations, North Holland Math. Stud. Elsivier, Amsterdam. 204 (2006).

[23] V. Kiryakova, The special functions of fractional calculus as generalized fractional calculus operators of some basic functions, Computers and Mathematics with applications. 59 (2010). 1128-1141.

[24] V. Lakshmikantham, S. Leela, J. Vasundhara, Theory of fractional dynamic systems, Cambridge Scientific Publishers, Cambridge. (2009).

[25] V. Lakshminarayanan, L.S. Varadharajan, Special functions for optical science and engineering, SPIE. (2015).

[26] L.D. Landau, E.M. Lifshitz, Quantum mechanics: non-relativistic theory, Institute of physical problems, U.S.S.R. Academy of Sciences. (1965).

[27] A. Loverro, Fractional calculus: History, definitions and applications for the engineer, Institute of physical problems, U.S.S.R. Academy of Sciences. (2004).

[28] F. Mainardi, A. Mura and G. Pagnini, The M-Wright function in time-fractional diffusion processes: a tutorial survey, International Journal of Differential Equations. 2010.

[29] H. Mohammadi, S. Etemad, A theoretical study of the Caputo-Fabrizio fractional modeling for hearing loss due to Mumps virus with optimal control, Chaos, Solitons and Fractals. 144 (2021) 110668.

[30] M.D Ovidio, E. Orsingher and B. Toaldo, Time-chenged processes governed by space-time fractional telegraph equations, Math. PR. (2013).

[31] S. Pitts, Mean-square fractional calculus and some applications, Scool of Mathematics, Statistics and Computer Science University of Kwazulu-Natal. (2012).

[32] A. Salim, M. Benchohra, J. E. Lazreg, J. Henderson, Nonlinear implicit generalized Hilfer-type fractional differential equations with non-instantaneous impulses in Banach spaces, ATNAA journal. 4(4) (2020) 332-348.

[33] V.E. Tarasov, Mathematical economics: application of fractional calculus, Mathematics. 8(5) (2020) 660.

[34] S.T.M. Thabet, S. Etemad, S. Rezapour, On a coupled Caputo conformable system of pantograph problems, Turkish Journal of Mathematics. 45(1) (2021) 496-519.

[35] O. Vallee, M. Soares, Airy functions and applications to physics, Imperial College Press, London. (2010).

[36] A. Vinodkumar, K. Malar, M. Gowrisankar, P. Mohankumar, Existence, uniqueness and stability of random impulsive fractional differential equations, Acta Mathematica Scientia. 36(2) (2016) 428-442.

[37] H. A. Wahash, M. Abdo, S. Panchal, Existence and stability of a nonlinear fractional differential equation involving a

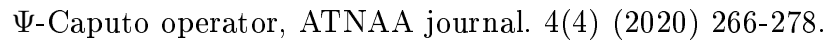

[38] H. Yfrah, Z. Dahmani, M.Z. Sarikaya and F.A. Gujar, A sequential nonlinear random fractional differential equation: existence, uniqueness and new data dependence. Submitted 2020.

[39] H. Yfrah, Z. Dahmani, L. Tabharit and A. Abdelenbi, High order random fractional differential equations: existence, uniqueness and data dependence, Journal of Interdisciplinary Mathematics. (2020) Accepted. 